\title{
Influence of the implanted pulse generator as reference electrode in finite element model of monopolar deep brain stimulation
}

\author{
Grégoire Walckiers $^{\mathrm{a}, *}$, Benjamin Fuchs ${ }^{\mathrm{a}, 1}$, Jean-Philippe Thiran ${ }^{\mathrm{b}, 2}$, Juan R Mosig ${ }^{\mathrm{a}, 3}$, Claudio Pollo ${ }^{\mathrm{b}, \mathrm{c}, 4}$ \\ a Electromagnetics and Acoustic Laboratory, Ecole Polytechnique Fédérale de Lausanne (EPFL), 1015 Lausanne, Switzerland. \\ b Signal Processing Institute, Ecole Polytechnique Fédérale de Lausanne, 1015 Lausanne, Switzerland. \\ ${ }^{\mathrm{c}}$ Neurosurgery Department, Centre Hospitalier Universitaire Vaudois (CHUV), 1011 Lausanne, Switzerland.
}

\section{A R T I C L E I N F O}

\section{Article history:}

Received 28 May 2009

Received in revised form 13 October 2009

Accepted 13 October 2009

\section{Keywords:}

Deep brain stimulation

Finite element

Volume of tissue activated

Diffusion tensor

Parkinson's disease

\begin{abstract}
A B S T R A C T
Electrical deep brain stimulation (DBS) is an efficient method to treat movement disorders. Many models of DBS, based mostly on finite elements, have recently been proposed to better understand the interaction between the electrical stimulation and the brain tissues. In monopolar DBS, clinically widely used, the implanted pulse generator (IPG) is used as reference electrode (RE).

In this paper, the influence of the RE model of monopolar DBS is investigated. For that purpose, a finite element model of the full electric loop including the head, the neck and the superior chest is used. Head, neck and superior chest are made of simple structures such as parallelepipeds and cylinders. The tissues surrounding the electrode are accurately modelled from data provided by the diffusion tensor magnetic resonance imaging (DT-MRI). Three different configurations of RE are compared with a commonly used model of reduced size.

The electrical impedance seen by the DBS system and the potential distribution are computed for each model. Moreover, axons are modelled to compute the area of tissue activated by stimulation.

Results show that these indicators are influenced by the surface and position of the RE. The use of a RE model corresponding to the implanted device rather than the usually simplified model leads to an increase of the system impedance $(+48 \%)$ and a reduction of the area of activated tissue $(-15 \%)$.
\end{abstract}

(c) 2009 Elsevier B.V. All rights reserved.

\section{Introduction}

High frequency deep brain stimulation (DBS) of the subthalamic nucleus (STN) is a surgical technique which has proved during recent years its ability to improve symptoms in Parkinson's disease (Benabid et al., 1996; Vingerhoets FJG et al., 2002; Rodriguez-Oroz et al., 2005; Wider et al., 2008). Recent studies also suggested that STN DBS could be useful in the treatment of epilepsy in animal mod-

* Corresponding author at: EPFL STI LEMA, ELB Station 11, CH-1015 Lausanne, Switzerland. Tel.: +4121693 4637; fax: +41216932673.

E-mail addresses: gregoire.walckiers@a3.epfl.ch (G. Walckiers),

benjamin.fuchs@univ-rennes1.fr (B. Fuchs), jean-philippe.thiran@epfl.ch

(J.-P. Thiran), juan.mosig@epfl.ch (J.R. Mosig), Claudio.Pollo@chuv.ch (C. Pollo).

${ }^{1}$ Address: Universit de Rennes 1, IETR-UMR 6164, Bat. 11D, Campus de Beaulieu, 263 Avenue Gnral Leclerc, F-35042 RENNES Cedex, France. Tel.: +33 22323 6731; fax: +33223236969.

${ }^{2}$ Address: EPFL STI IEL LTS5, ELD Station 11, CH-1015 Lausanne, Switzerland. Tel.: +4121693 4623; fax: +41216937600.

3 Address: EPFL STI LEMA, ELB Station 11, CH-1015 Lausanne, Switzerland. Tel.: +4121693 4628; fax: +41216932673.

${ }^{4}$ Address: Neurochirurgie BH-13, Centre Hospitalier Universitaire Vaudois (CHUV), Bugnon 46, CH-1011 Lausanne, Switzerland. Tel.: +41 21314 2551; fax: +41 213142605 . els (Usui et al., 2005) as well as in human (Chabardes et al., 2002; Lee et al., 2006). It consists of delivering electrical pulses within the STN through an implanted electrode connected to an implanted pulse generator (IPG) (Pollo et al., 2007). Despite its clinical efficiency, the mechanisms of action underlying STN DBS as well as the volume of tissue around the electrode influenced by the electrical current remain debated (McIntyre and Thakor, 2002; McIntyre et al., 2004b,c).

To better understand the mechanisms involved in STN DBS, numerous computational finite element (FE) models of electrical potentials released from a determined source have been proposed (Butson and McIntyre, 2005, 2006; Butson et al., 2006, 2007; McIntyre et al., 2004a, 2007). They have shown the importance of various parameters such as: the electrode geometry (Butson and McIntyre, 2006; Butson et al., 2006; Wei and Grill, 2005), the electric pulse amplitude and duration (Butson and McIntyre, 2007), the tissue-electrode capacitance (Butson and McIntyre, 2005) and the peri-electrode space (Yousif et al., 2007) on the electrical potential distributions. The influence of electrical properties of surrounding brain tissues, namely the conductivity and the anisotropy, described by diffusion tensor magnetic resonance imaging (DTMRI), has also been investigated (McIntyre et al., 2004a; Butson et al., 2007; Sotiropoulos and Steinmetz, 2007). However, most of 


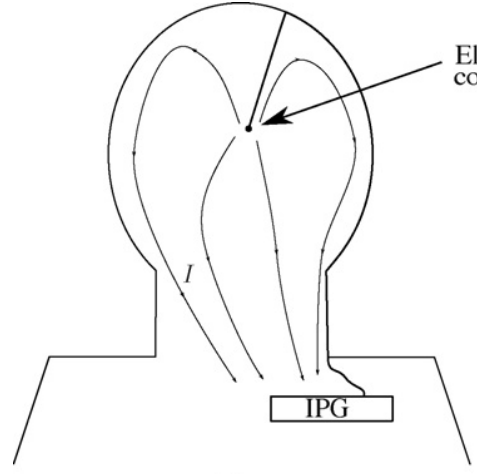

(a)
Electrode

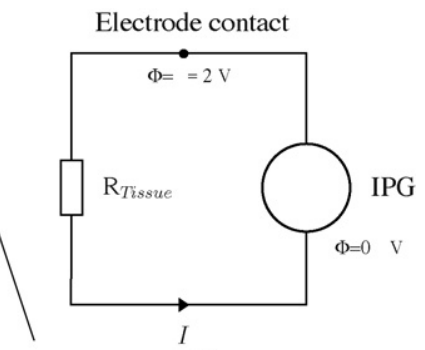

(b)
Fig. 1. Schematic views of the electric loop involved in monopolar stimulation. The implanted system is represented in (a). The current I emitted by the electrode contact reaches the IPG through the tissues. The reference potential is set on the IPG case. An implanted wire (bold) links the electrode contact to the IPG. The electric equivalent circuit is depicted in (b).

the previous studies have used models of reduced size and/or simplified electrical properties of brain tissues for computation load reasons (Butson and McIntyre, 2005, 2006; Butson et al., 2006, 2007; McIntyre et al., 2004a; Walckiers et al., 2007; Yousif et al., 2007).

In these models, a zero potential is usually applied on the external boundary to set up the reference electrode (RE) in case of monopolar stimulation. Therefore, only one part of the electric loop is considered. Indeed, the entire electrical loop involved in monopolar STN DBS includes all the biological tissues between the electrode active contact and the RE, i.e. the IPG, as shown in Fig. 1. The accuracy of the electrical potential distribution around the electrode can then be questioned when modelling monopolar DBS with such approximated models. The aim of this study is to evaluate the influence of such a simplification.

In this paper, a FE model of the whole electric loop involved in monopolar STN DBS is proposed. This model encompasses the head, the neck and the upper chest. Head, neck and superior chest are made of simple structures such as parallelepipeds and cylinders. The conductivity of the tissues crossed by the electric loop is also considered. Special care is taken to model the electrical anisotropic properties of the tissues surrounding the electrode. These properties come from DT-MRI data of a patient before surgery.

A realistic model of the $R E$ is built. Its size and position are based on what is used in clinical applications. The influence of the RE surface and position on the potential distribution is studied. Three full loop model results are compared with a commonly used model of reduced size that is considered as the reference.

Moreover, the response of axons to the potential distribution is investigated using a typically used axon model (Butson and McIntyre, 2005, 2006; Butson et al., 2006; McIntyre et al., 2002; Sotiropoulos and Steinmetz, 2007). The impact of the RE models on the tissue activated by the stimulation is evaluated and discussed.

\section{Methods}

The electrical parameters of STN DBS currently used in clinical practice to treat Parkinson's disease patients are the following: a frequency of $130-180 \mathrm{~Hz}$ and a pulse width of $60-120 \mu \mathrm{s}$. In this frequency range, the potential distribution can be considered as a quasistatic bioelectric problem (Plonsey and Heppner, 1967). The potentials can then be found by solving the Poisson equation $\nabla$. $\overline{\bar{\sigma}} \nabla \Phi=0$, where $\nabla$ is the standard differential operator "del", $\overline{\bar{\sigma}}$ describes the known tensorial conductivity of the medium and $\Phi$ is the electric potential (Broek et al., 1996; Miller and Henriquez, 1990). Once the electric potentials are obtained, the electric field $\bar{E}$ can be deduced since $\bar{E}=-\nabla \Phi$. The currents $\bar{J}$ can finally be derived from $\bar{E}$ using $\bar{J}=\overline{\bar{\sigma}} \bar{E}$.

In this study, the considered electric quantity is the electric potential $\Phi$ since, according to Rattay's study (Rattay, 1989), the activation of a neuron by an extracellular stimulation is linked to the double spatial derivative of the external potential along the fiber direction, $\partial^{2} \Phi / \partial^{2} x$. The integration of the current density vector $\bar{J}$ around the electrode provides the total current $I$ (in A) released during stimulation. The potential on the electrode contact is fixed through a Dirichlet type of boundary condition to $-2 \mathrm{~V}$. The impedance of the tissues $R_{\text {tissue }}$ crossed by the electric loop is computed using $R=\Delta \Phi / I$, where $\Delta \Phi$ is the fixed potential difference between the electrode contact and the RE (Fig. 1b).

\subsection{Model geometry}

The FE model is composed of the upper part of the chest, the neck and the head. The geometry of the model is made of simple structures (parallelepipeds and cylinders, see Fig. 2a). The head and the chest are made of parallelepipeds. The spine is modelled by two concentric cylinders (spinal cord surrounded by bone). The gray and white matter are separated into two hemispheres surrounded by cerebrospinal fluid (CSF) and a skull. The rest of the model is essentially made of muscle. Fig. 2 b shows a two dimensional map of the tissues used in the model. The use of this simplified geometries

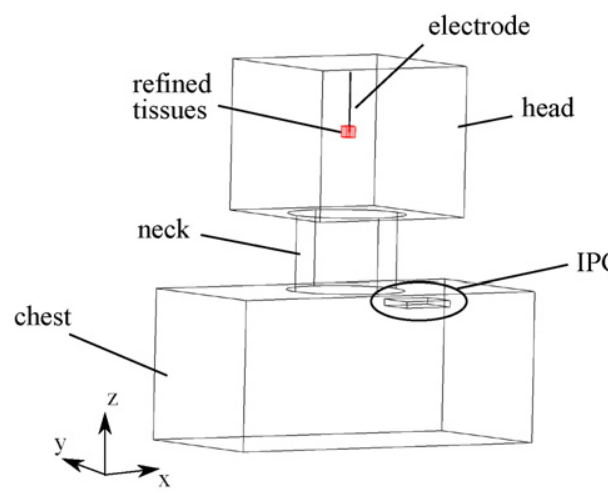

(a)

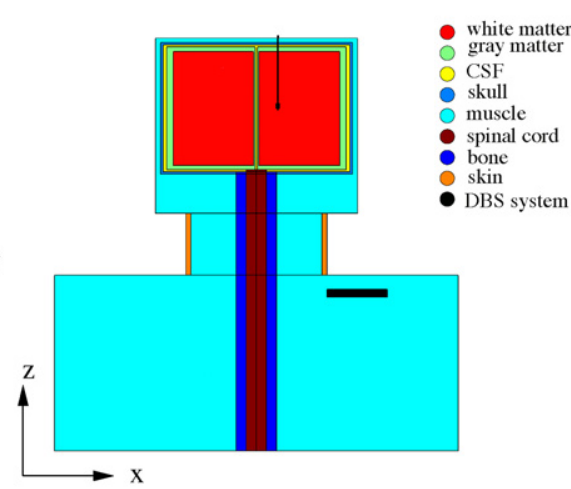

(b)

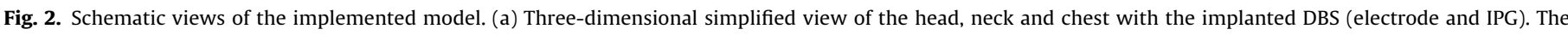

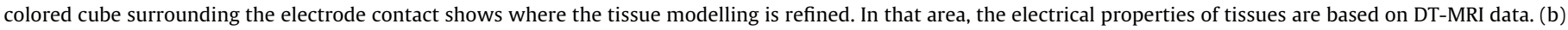
Cutting view of the FE model with the biological tissue characteristics. 


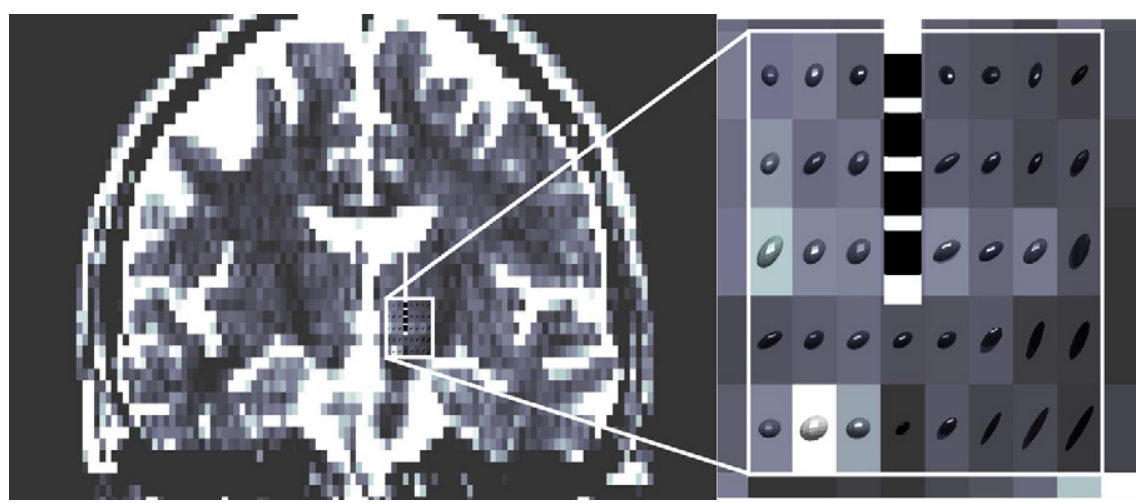

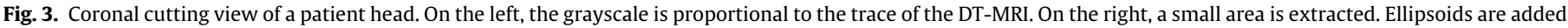

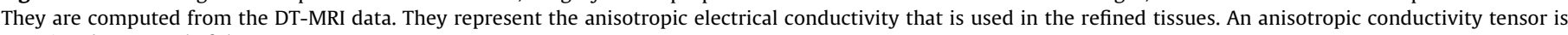
associated to a voxel of the DT-MRI.

aims to reduce the number of unknowns and to simplify the model generation.

On the external boundaries of the model, a null Neumann boundary condition (electrical insulation) is applied. This means that no currents are going through these surfaces. The model is made of approximately 400,000 nodes. The model mesh has been build with the help of COMSOL 3.3 (COMSOL Inc., Burlington, MA, USA). The mesh density has been forced to be high in the vicinity of the stimulation electrode, as the tetrahedral elements have edges of maximum $0.2 \mathrm{~mm}$. The solver used quadratic basis functions and the linear equation was solved with an algebraic multigrid preconditioned conjugate gradient solver. The Dirichlet boundary conditions are embedded in the equation system with an elimination technique.

A more realistic geometrical representation of the human head was presented in Wolters et al. (2006). However, our FE model, with simple structure geometries and a reasonable mesh density, might already provide reasonable results for the quantities under study. Even if first examinations with regard to numerical convergence for a similar potential problem in FE head volume conductor models has been carried out (Wolters et al., 2007), a completely satisfying qualitative convergence proof for a problem with discontinuous tissue conductivities is difficult and has not yet been achieved. We have however checked that doubling the node number changes only the potential values by amounts always smaller than $1.75 \%$.

\subsection{Electrical properties of tissues}

The model is sampled in eight classes of tissues (see Fig. 2b), having their own fixed isotropic electrical conductivity (in $\mathrm{S} \mathrm{m}^{-1}$ ): muscle (0.1), skin (0.434), bone (0.04), skull (0.0063), spinal cord (0.154), CSF (1.538), white (0.143) and gray (0.33) matter. Theses values of conductivity are taken from Haueisen et al. (1997).

Previous studies have shown that both the electrical conductivity and anisotropy of the tissues in the vicinity of the source have a major impact on the potential distribution generated by the stimulation (Haueisen et al., 1997, 2000; Wolters et al., 2005, 2006). It is therefore crucial to have an accurate model in this region. To increase the quality of our model, the tissue conductivities surrounding the electrode contact are refined in a box of $12 \mathrm{~mm} \times 12 \mathrm{~mm} \times 15 \mathrm{~mm}$ (see Fig. 2a). The anisotropic electrical conductivity of each element in that box is based on the values of water diffusion tensor $\overline{\bar{d}}$ provided by a high resolution DT-MRI dataset (voxels of $1.5 \mathrm{~mm} \times 1.5 \mathrm{~mm} \times 3 \mathrm{~mm}$ ) taken from a patient before surgery. This DT-MRI dataset was based on a single-shot EPI sequence $(\mathrm{TR} / \mathrm{TE}=1000 / 89 \mathrm{~ms}, 44$ contiguous slices of $3 \mathrm{~mm}$ of thickness, FoV $=210 \mathrm{~mm} \times 210 \mathrm{~mm}$, matrix size $128 \times 128$ ) acquired at $b$-value $=1000 \mathrm{~s} / \mathrm{mm}^{2}$ along 6 diffusion sensitizing directions placed with a sagittal symmetry and forming the vertices of an icosahedron. As the voxels are taken in the center of the image, no corrections for the distortion have been applied. The targeted position of the electrode in the DT-MRI is set by a clinician. For Parkinson's disease treatment, this target is located in the subthalamic nucleus. Each element in this box has an anisotropic electrical conductivity computed from the water-diffusion tensor (Fig. 3)

The study of Tuch et al. (2001) has shown a linear relation between the water diffusion and the electrical transport through cerebral tissues. This linear model has been successfully validated by $\mathrm{Oh}$ et al. (2006). In this linear model, a scaling parameter between water diffusion tensor $\overline{\bar{d}}$ and electrical conductivity tensor $\overline{\bar{\sigma}}$ has to be chosen. In this study, stimulated by the "volume constraint" in the simulation study of Wolters et al. (2006), a new approach is used. Each water diffusion tensor is scaled according to Eq. 1 in order to achieve the electrical conductivity tensor at the same position in space:

$\overline{\bar{\sigma}}_{\text {electric }}=\overline{\bar{d}}_{\text {DTMRI }} \cdot\left(\frac{V\left(\overline{\bar{\sigma}}_{\text {CSF }}\right)}{V\left(\overline{\bar{d}}_{\text {DTMRI }}\right)_{\max }}\right)^{1 / 3}$,

where $V()$ is the volume of an ellipsoid representing either the tensor $\overline{\bar{\sigma}}$ or $\overline{\bar{d}}$, and $V()_{\max }$ denotes the maximum volume value. This operation first normalizes the ellipsoid volume with respect to the biggest volume obtained from DT-MRI. Then, as the CSF is the tissue that has the highest electrical conductivity, the ellipsoids are scaled by the volume of an isotropic conductivity tensor of CSF.

\subsection{Reference electrode models}

In monopolar stimulation, the RE of the electric loop is the IPG. The IPG is modelled as a box of $6 \mathrm{~cm} \times 5 \mathrm{~cm} \times 1 \mathrm{~cm}$. These dimensions correspond to the Soletra system of Medtronic (Soletra 7426, Medtronic, Minneapolis, MN, USA).

Four RE models are investigated. They present various type of boundary conditions (BC), as depicted in Fig. 4:

- BC I: the IPG is located in the chest. One of the main faces $\left(30 \mathrm{~cm}^{2}\right)$ of the box is set to the reference potential $(0 \mathrm{~V})$ while the other faces are considered as isolating surfaces (no current flow through the surfaces).

- BC II: the IPG is located in the chest and has its two main faces set to $0 \mathrm{~V}$. The surface of the RE is then doubled $\left(60 \mathrm{~cm}^{2}\right)$.

- BC III: the IPG is located in the bottom of the head, closer to the electrode (see Fig. 4), with one face set to $0 \mathrm{~V}$. 
BOUNDARY CONDITIONS

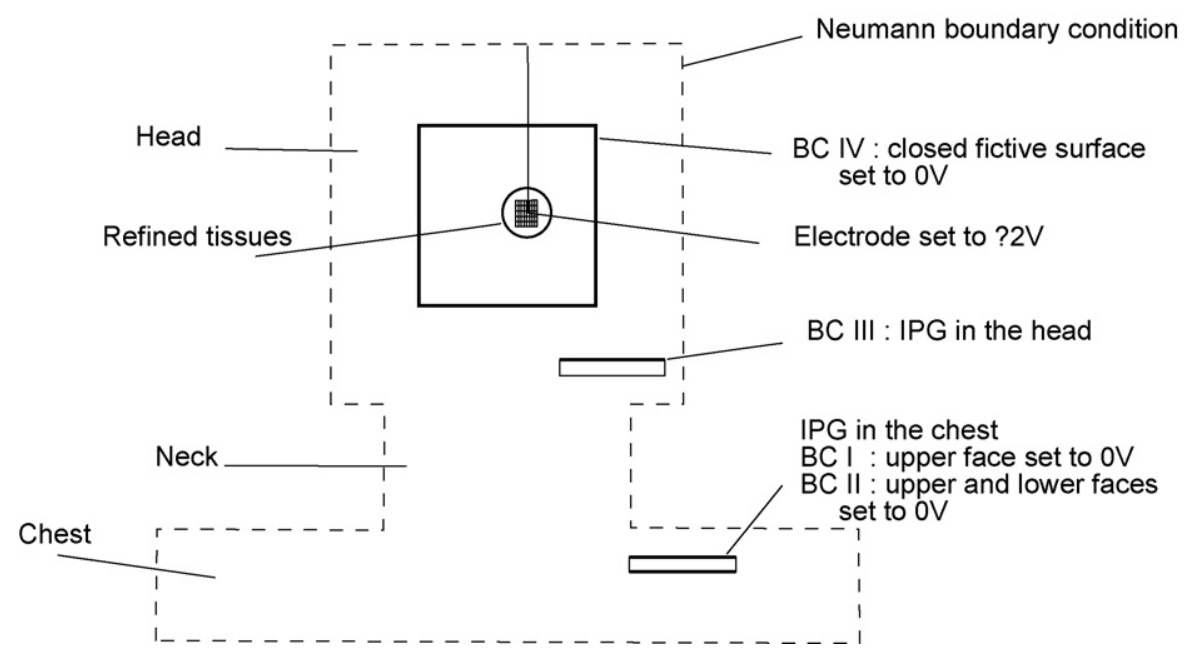

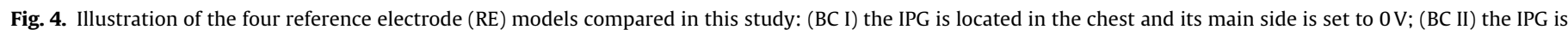

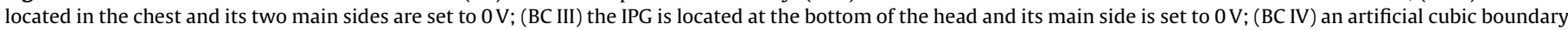

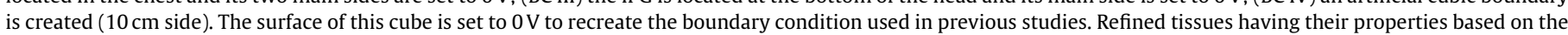
DT-MRI can be seen in the center of the head.

- BC IV: the surface of a $10 \mathrm{~cm}$ side cube $\left(600 \mathrm{~cm}^{2}\right)$ surrounding the stimulation electrode is set to $0 \mathrm{~V}$.

The area and location of the boundaries of the BC I model correspond to the implanted system. $\mathrm{BC}$ IV, which uses the same approximation than the previous studies models (Butson and McIntyre, 2005, 2006; Butson et al., 2006; McIntyre et al., 2004a; Sotiropoulos and Steinmetz, 2007; Yousif et al., 2007), is considered as the "control model" to ascertain the improvements introduced by our full loop models. Indeed, these studies have modelled the RE on the surface of their reduced size models. The potential on this surface was set to $0 \mathrm{~V}$.

\subsection{Electrode contact geometry}

The electrode contact model is based on the DBS electrode manufactured by Medtronic (ACTIVA 3389-Medtronic, Minneapolis, MN, USA). It consists of four cylinders of $1.27 \mathrm{~mm}$ diameter and $1.5 \mathrm{~mm}$ length of conductive material separated by cylinders of the same diameter and of $0.5 \mathrm{~mm}$ height of isolating material, as already used in previous studies (Butson and McIntyre, 2006; Wei and Grill, 2005). An illustration is shown if Fig. 6. In contrary to the manufac- tured device, the tip of the electrode is not curved. Nevertheless, the electric conductivity applied to this volume is very low, as it is an insulator, and the influence of neglecting the curvature of the tip on the potential distribution around the active electrode is likely to be negligible. Monopolar voltage controlled stimulation is often used clinically. In accordance to the standard clinical practice, $\mathrm{a}-2 \mathrm{~V}$ fixed voltage (Dirichlet boundary condition) is set for all the simulations on the more distal contact (the lowest cylinder in Fig. 6).

\subsection{Neural activation prediction}

The steps to evaluate the neural response to stimulation are summarized in Fig. 5. The NEURON environment tool (Hines and Carnevale, 2001) is used to model the axons as already done in previous studies (Butson and McIntyre, 2005, 2006; Butson et al., 2006; McIntyre et al., 2002; Sotiropoulos and Steinmetz, 2007). With this tool, models of $5.7 \mu \mathrm{m}$-diameter myelinated axons made of 20 segments and 21 nodes are created. The detailed axon's model structure can be found in (Butson and McIntyre, 2006; McIntyre et al., 2002).

The value of the extracellular potential at each node is interpolated from the results given by the FE model. A time-varying

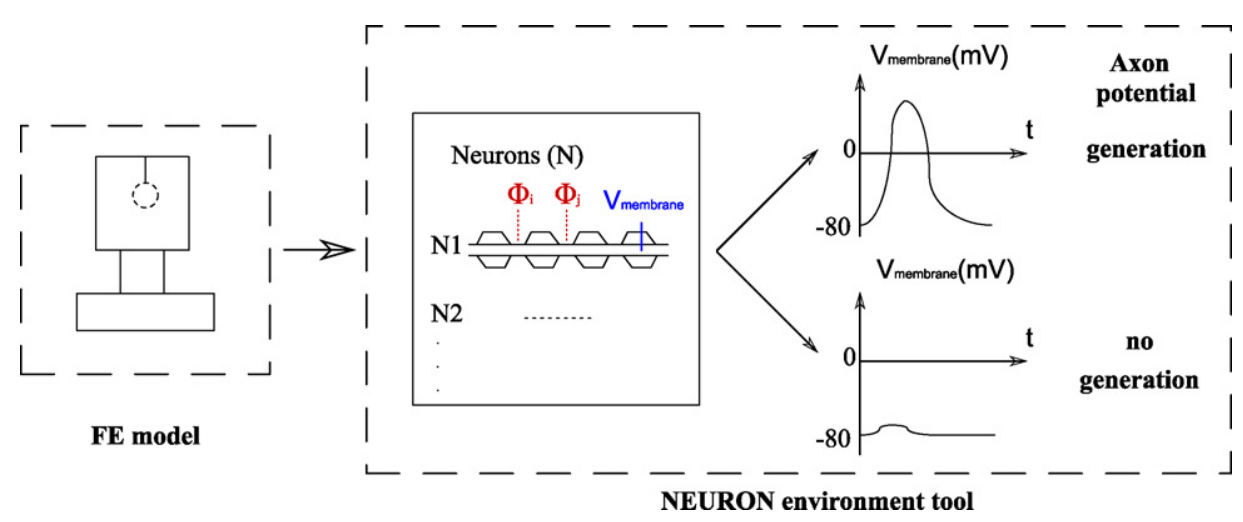

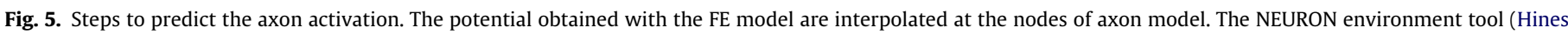

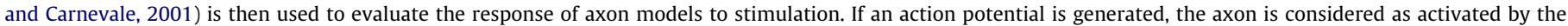
stimulation. 


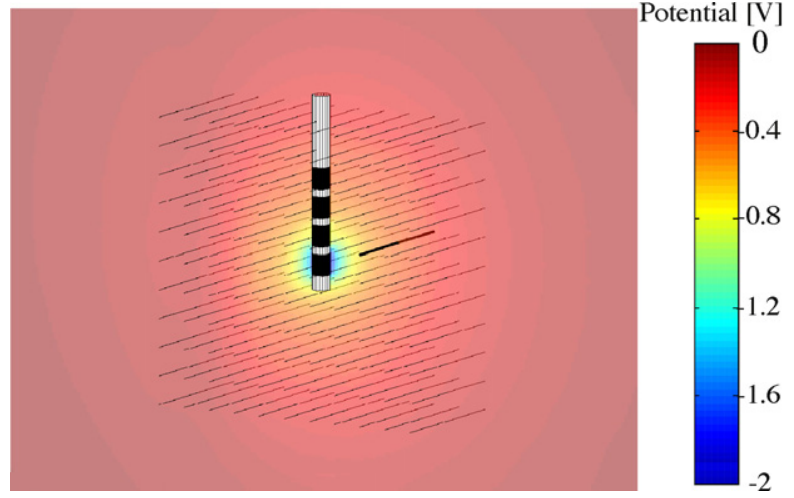

Fig. 6. Positions of the axon models used to evaluate the activation of tissue. The axon models are perpendicular to a plane containing the electrode shaft. The extracellular potentials are interpolated from the values of potential obtained from the FE model.

Table 1

RE model influence on the impedance, the potential distribution.

\begin{tabular}{lrrl}
\hline RE model & $R_{\text {tissue }}[\Omega]$ & $\begin{array}{l}R_{\text {tissue relative }} \\
\text { error [\%] }\end{array}$ & $\begin{array}{l}\text { Volume of tissue } \\
{\left[\mathrm{mm}^{3}\right] \text { such as }} \\
-2<\Phi<-1 \mathrm{~V}\end{array}$ \\
\hline BC I & 1001 & +48 & 272 \\
BC II & 953 & +40 & 160 \\
BC III & 853 & +27 & 49 \\
BC IV & 674 & 0 & 19 \\
\hline
\end{tabular}

electric field is created convoluting the obtained potentials by a single normalized electric pulse time course (duration $5 \mathrm{~ms}$ with a pulsewidth of $200 \mu \mathrm{s}$ ). These values are out of the usual range of stimulation, nevertheless it has no impact on the purpose of this study which is to show the influence of the boundary conditions. The axon response to stimulation is only computed for axons perpendicular to the electrode and having their centers on a plane containing the electrode shaft (see Fig. 6). This choice of axon model and direction is based on the approach used in previous studies. A simple integration scheme is used to compute the size of the surface activated, summing the discretized areas where an activation of a $1: 1$ ratio takes place. The equivalent radius of this surface is then extracted to provide the mean extend of the stimulation.

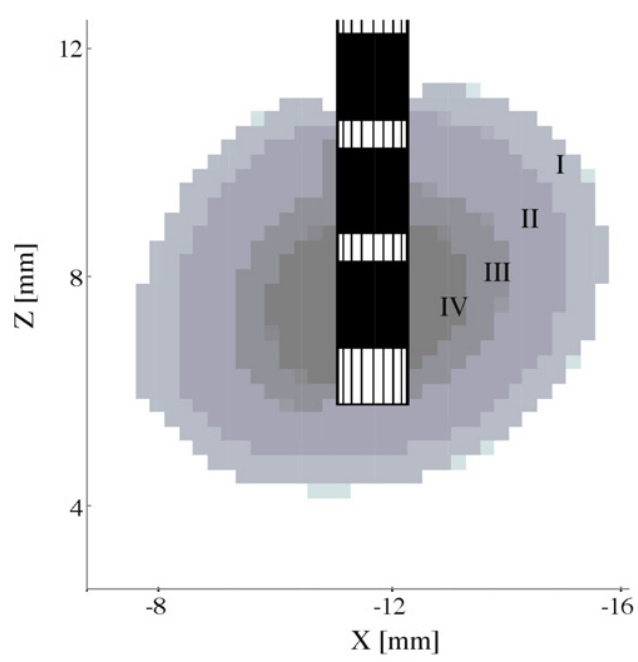

Fig. 7. Isopotentials of half the pulse amplitude $(-1 \mathrm{~V}$ here) obtained using four reference electrode (RE) models (I stands for BC I). The potentials generated by the stimulation reach more rapidly the value of $-1 \mathrm{~V}$ when the surface set to $0 \mathrm{~V}$ is closer (IV, III) or larger (II) than when using the most realistic model for the RE (I).
Table 2

RE model influence the predicted activation of tissues.

\begin{tabular}{lll}
\hline RE model & Surface activated $\left[\mathrm{mm}^{2}\right]$ & Equivalent radius $[\mathrm{mm}]$ \\
\hline BC I & 34 & 3.3 \\
BC II & 36 & 3.4 \\
BC III & 41 & 3.6 \\
BC IV & 48 & 3.9 \\
\hline
\end{tabular}

\section{Results}

\subsection{Influence of the RE model on the impedance and potential distribution}

The evaluated impedance of the tissues located between the electrode contact and the RE in monopolar stimulation are reported in Table 1 for the four RE models. The computed volume of tissue such as the potential $\Phi$ ranges from -2 to $-1 \mathrm{~V}$ (half value of the electric pulse) is also given.

The highest value of impedance is obtained with the the RE model BC I (1001 $\Omega$ ). For this case, we observe an increase of the tissue impedance of $48 \%$ compared to the reference boundary condition model (BC IV). Doubling the surface of the RE (BC II) induces a small decrease of the impedance $(953 \Omega)$. When moving the RE to the base of the head, the impedance reaches $853 \Omega$. Finally, using the boundary condition BC IV yields to an impedance value of 674 $\Omega$.

The potential distribution is also affected by the RE model, as exposed in Fig. 7. Isopotentials of $-1 \mathrm{~V}$ (half pulse amplitude) are shown. The potentials generated by the stimulation reach more rapidly the value of $-1 \mathrm{~V}$ when the $0 \mathrm{~V}$ surface is closer to the electrode contact and/or larger.

\subsection{Influence of the RE model on the tissue activation}

The response of axon models to stimulation has also been evaluated for the four RE models. When the impedance value decreases, the number of modelled axons where an action potential takes place increases as shown in Fig. 8. The obtained area of stimulated axons and their equivalent radius are shown in Table 2. The stimulation spreads up to $3.3 \mathrm{~mm}$ from the electrode contact surface when using BC I (see Fig. 8a). BC IV leads to an activation of the maximum number of axons, which spreads up to $3.9 \mathrm{~mm}$ away from the electrode contact (Fig. 8d).

\section{Discussion}

Predicting as accurately as possible the volume of tissue influenced by the electrical current around the electrode is crucial to better understand the mechanisms involved in DBS. This is a particularly important consideration in STN DBS, where the mechanisms of action underlying electrical stimulation are still under evaluation (McIntyre and Thakor, 2002; McIntyre et al., 2004b,c). The structure(s) influenced by DBS is(are) a matter of debate as, not only the STN neurons themselves, but also the axons surrounding the STN may play an important role in the improvement of the symptoms of the disease.

Pioneering work has been performed by the McIntyre's group in the field of DBS modelling and prediction of the volume of tissue influenced by electrical stimulation using FE method (Butson and McIntyre, 2005, 2006, 2007; Butson et al., 2006, 2007; McIntyre et al., 2004a, 2007). Based on the assumption that the reference potential is estimated to be far enough from the electrode contact, they apply a $0 \mathrm{~V}$ boundary condition on the boundary of their model limited to a restricted area of the head (Butson et al., 2006; Butson and McIntyre, 2005, 2006, 2007; McIntyre et al., 2004a; 


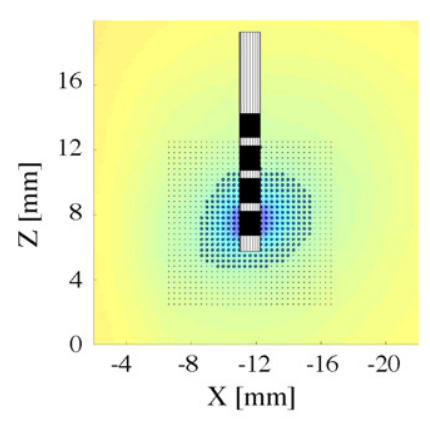

(a) BC I

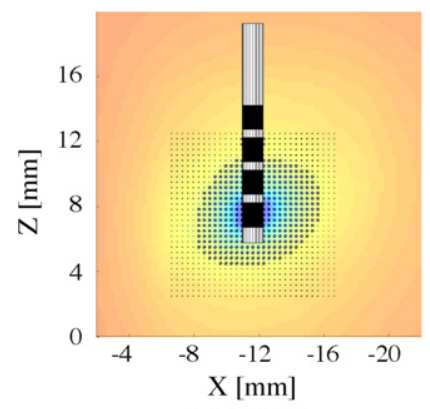

(c) BC III

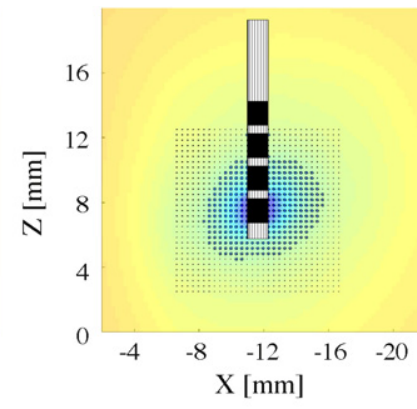

(b) BC II

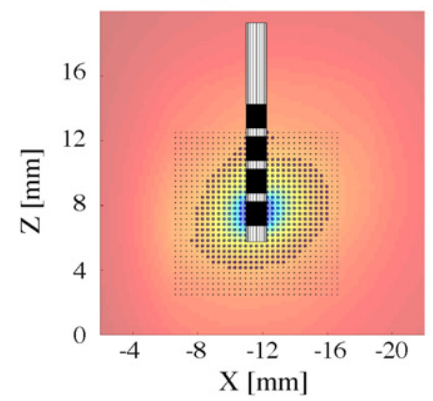

(d) BC IV

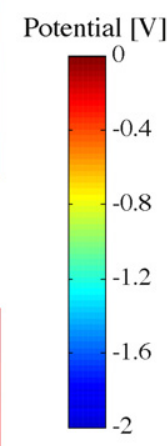

Action potential

- generation no generation

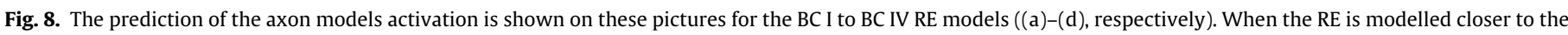
electrode contact, an increase of the area of stimulated axons is observed. The use of BC IV leads to the maximal area of stimulated axons.

Yousif et al., 2007). In bipolar stimulation, as the electric loop goes from one active contact to the other, the IPG influence can be neglected and the assumption seems fully justified. However, in the case of monopolar stimulation, most often used in clinical practice (Volkmann et al., 2002), this assumption may be questioned as the electric loop goes indeed from the electrode contact to the IPG located in the superior chest. All the tissues crossed by the electrical current have an impact on the total impedance, and consequently on the current and charges released by the stimulation.

The model's geometry used in this study is simplified in order to avoid the huge preprocessing steps needed to build a model fully based on anatomical data. Although using such fully realistic model would probably induce some variations on the prediction of tissue activated by the stimulation, the effect of boundary conditions applied would remain unchanged. The sensitivity of the obtained potentials with regards to the volume conductor misspecifications has to be examined in a later study. Nevertheless, despite the simplifications performed on our model geometry, we were able to obtain a total impedance of tissues between the active contact and the IPG of approximately $1 \mathrm{k} \Omega$. This value is in accordance with the measurements performed in patients having implanted systems (Volkmann et al., 2002). The impact of the tissues anisotropy could also be evaluated in further studies, but then a special care should be taken to choose the equivalent isotropic conductivity. Several approaches are possible such as using a segmentation process to assign to each voxel a type of tissue and a corresponding conductivity. One could also assign to each voxel's conductivity the mean, or the maximum of the eigenvalues of the obtained conductivity tensors.

The results of this study show that doubling the surface set to the reference potential slightly reduces the impedance. Applying the reference potential to the same surface but in a position closer to the electrode contact leads to a reduction of the impedance, as less tissues are crossed by the current to close the electric loop. Using the RE model based on the DBS device (RC I), instead of the usual boundary condition applied in previous studies (RC IV), where $0 \mathrm{~V}$ is set on the surface of a $10 \mathrm{~cm}$ cube enclosing the electrode, leads to an increase of $48 \%$ of the tissue impedance. The potential distribution also reflects these changes of impedance as shown in Fig. 7. A smaller global tissue impedance induces a reduction of the volume of tissue delimited by the half pulse amplitude isopotential $(-1 \mathrm{~V})$.

The evaluation of the stimulation on axon models provides an idea of the volume of tissue activated in the plane orthogonal to the axon models. But this area is only a basic indicator on the effect of stimulation. Indeed, the assumption that all the axons are perpendicular to the same plane and share the same physiological structure is far from reality. The properties of the axons model, myelinated fibers having their directions perpendicular to the electrode shaft, are based on the models used in the previous studies (Butson et al., 2006; Butson and McIntyre, 2005, 2006; McIntyre et al., 2002; Sotiropoulos and Steinmetz, 2007). The use of soma models and interconnected neurons rather than isolated ones should be studied in a further work. The obtained areas show that the axons are activated up to a mean distance of $3.9 \mathrm{~mm}$ which is in agreements with what is observed in previous modeling studies (Butson et al., 2006, 2007; Butson and McIntyre, 2005, 2006, 2007; McIntyre et al., 2004a; Sotiropoulos and Steinmetz, 2007). Using a RE model corresponding to the implanted system (a full loop), rather than the boundary condition applied in previous studies, reduces by approximately $15 \%$ (equivalent radius of $3.3 \mathrm{~mm}$ ) the mean spread of the predicted stimulated area, as shown in Fig. 9. This modification is not negligible in STN DBS. Indeed, clinical observations have shown that switching the stimulation from one contact to the adjacent one (shift of $2 \mathrm{~mm}$ ) can lead to an important reduction of the benefits of stimulation and to the onset of side effects. Moreover, increasing the quality of the prediction of tissue activation for each patient presents some major interests. It provides pre-surgical information on the best localization and stimulation parameters to maximize the benefits of the stimulation and reduce side effects. It also gives post-surgical information on the activated area involved in long term effects of DBS.

The model proposed in this study is based on real data coming from a patient DT-MRI before surgery. Modeling a realistic RE leads to a non-negligible reduction of the area of tissue activated. To go further, more accurate results could be obtained using the 


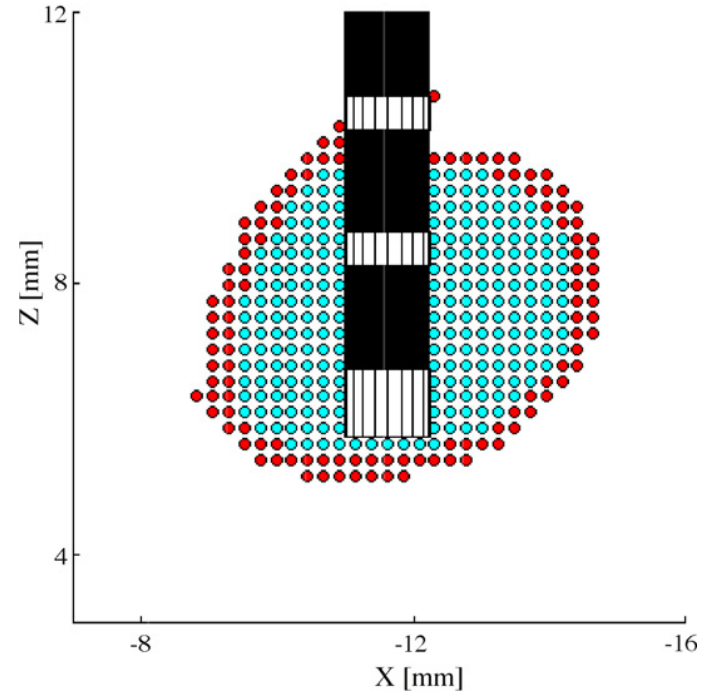

Fig. 9. The predicted activation of tissues obtained using boundary conditions corresponding to the implanted system (in cyan) is smaller than when using the boundary conditions of a reduced size model (in red). (For interpretation of the references to color in this figure legend, the reader is referred to the web version of the article.)

definitive localization of the electrode and including the electrode brain interface, which has been shown to impact the potential distribution (Yousif et al., 2007). Indeed it has been shown that including a capacitive model of the electrode tissue interface would have reduced even more the area of tissue activated (Butson and McIntyre, 2005).

In this study, we have considered a voltage controlled stimulation. In future investigations, it would be interesting to use a current controlled stimulation in order to avoid the encountered problem of the ground position and surface. Indeed, in that case, the total current issued from the electrode contact is equal to the current going out of the boundaries of the reduced size model. However, let us note that the values of the tissue conductivity (that are not precisely known) will have a major impact on the potentials created by the stimulation.

\section{Acknowledgments}

This study was supported by research grants from the European Society of Stereotactic and Functional Neurosurgery (ESSFN) and from the Swiss National Science Foundation (Grant 108318).

\section{References}

Benabid AL, Pollak P, Gao D, Hoffmann D, Limousin P, Gay E, et al. Chronic electrical stimulation of the ventralis intermedius nucleus of the thalamus as a treatment of movement disorders. J Neurosurg 1996;82:203-14.

Broek SP, Van Den, Zhou H, Peters MJ. Computation of neuromagnetic fields using finite-element method and Biot-Savart law. Med Bio Eng Comp 1996;34:21-6.

Butson CR, McIntyre CC. Tissue and electrode capacitance reduce neural activation volumes during deep brain stimulation. Clin Neurophysiol 2005;116:2490500 .

Butson CR, McIntyre CC. Role of electrode design on the volume of tissue activated during deep brain stimulation. J Neural Eng 2006;3:1-8.

Butson CR, McIntyre CC. Differences among implanted pulse generator waveforms cause variations in the neural response to deep brain stimulation. Clin Neurophysiol 2007;118:1889-94.

Butson CR, Maks CB, McIntyre CC. Sources and effects of electrode impedance during deep brain stimulation. Clin Neurophysiol 2006;117:447-54.

Butson CR, Cooper SE, Henderson JM, McIntyre CC. Patient-specific analysis of the volume of tissue activated during deep brain stimulation. Neuroimage 2007;34:661-70.
Chabardes S, Kahane P, Minotti L, Koudsie A, Hirsch E, Benabid AL. Deep brain stimulation in epilepsy with particular reference to the subthalamic nucleus. Epileptic Disord 2002;4(Suppl. 3):83-S93.

Haueisen J, Ramon C, Brauer H, Nowak H. The influence of local tissue conductivity changes on the magnetoencephalogram and the electroencephalogram. Biomed Tech (Berl) 2000;45:211-4.

Haueisen J, Ramon C, Eiselt M, Brauer H, Nowak H. Influence of tissue resistivities on neuromagnetic fields and electric potentials studied with a finite element model of the head. IEEE Trans Biomed Eng 1997;44:727-35.

Hines ML, Carnevale NT. NEURON: a tool for neuroscientists. Neuroscientist 2001;7:123-35.

Lee KJ, Jang KS, Shon YM. Chronic deep brain stimulation of subthalamic and anterior thalamic nuclei for controlling refractory partial epilepsy. Acta Neurochir Suppl 2006;99:87-91.

McIntyre CC, Thakor NV. Uncovering the mechanisms of deep brain stimulation for Parkinson's disease through functional imaging, neural recording, and neural modeling. Crit Rev Biomed Eng 2002;30:249-81.

McIntyre CC, Richardson AG, Grill WM. Modeling the excitability of mammalian nerve fibers: influence of afterpotentials on the recovery cycle. J Neurophysiol 2002;87:995-1006.

McIntyre CC, Mori S, Sherman DL, Thakor NV, Vitek JL. Electric field and stimulating influence generated by deep brain stimulation of the subthalamic nucleus. Clin Neurophysiol 2004a;115:589-95.

McIntyre CC, Savasta M, Goff L, Kerkerian-Le, Vitek JL. Uncovering the mechanism(s) of action of deep brain stimulation: activation, inhibition, or both. Clin Neurophysiol 2004b;115:1239-48.

McIntyre CC, Savasta M, Walter BL, Vitek JL. How does deep brain stimulation work? Present understanding and future questions. J Clin Neurophysiol 2004c;21:40-50.

McIntyre CC, Miocinovic S, Butson CR. Computational analysis of deep brain stimulation. Expert Rev Med Devices 2007;4:615-22.

Miller CE, Henriquez CS. Finite element analysis of bioelectric phenomena. Crit Rev Biomed Eng 1990;18:207-33.

Oh SH, Lee SY, Cho MH, Kim T-S, Kim IH. Electrical conductivity estimation from diffusion tensor and T2: a silk yarn phantom study. In: Proc Intl Soc Mag Reson Med; 2006.

Plonsey R, Heppner DB. Considerations of quasi-stationarity in electrophysiological systems. Bull Math Biophys 1967;29:657-64.

Pollo C, Vingerhoets FJG, Pralong E, Ghika J, Maeder P, Meuli, et al. Localization of electrodes in the subthalamic nucleus on magnetic resonance imaging. J Neurosurg 2007;106:36-44.

Rattay F. Analysis of models for extracellular fiber stimulation. IEEE Trans Biomed Eng 1989;36:676-82.

Rodriguez-Oroz MC, Obeso JA, Lang AE, Houeto J-L, Pollak P, Rehncrona S, et al Bilateral deep brain stimulation in Parkinson's disease: a multicentre study with 4 years follow-up. Brain 2005;128:2240-9.

Sotiropoulos SN, Steinmetz PN. Assessing the direct effects of deep brain stimulation using embedded axon models. J Neural Eng 2007;4:107-19.

Tuch DS, Wedeen VJ, Dale AM, George JS, Belliveau JW. Conductivity tensor mapping of the human brain using diffusion tensor MRI. PNAS 2001;98:11697-701.

Usui N, Maesawa S, Kajita Y, Endo O, Takebayashi S, Yoshida J. Suppression of secondary generalization of limbic seizures by stimulation of subthalamic nucleus in rats. J Neurosurg 2005;102:1122-9.

Vingerhoets FJG, Villemure J-G, Temperli P, Pollo C, Pralong E, Ghika J. Subthalamic DBS replaces levodopa in Parkinson's disease: two-year follow-up. Neurology 2002;58:396-401.

Volkmann J, Herzog J, Kopper F, Deuschl G. Introduction to the programming of deep brain stimulators. Mov Disord 2002;17(Suppl. 3):181-S187.

Walckiers G, Thiran J-P, Mosig JR, Pollo C. Iterative full head finite element model for deep brain stimulation. In: IEEE Proceedings 3rd International EMBS Conference on Neural Engineering; 2007.

Wei XF, Grill WM. Current density distributions, field distributions and impedance analysis of segmented deep brain stimulation electrodes. J Neural Eng 2005;2:139-47.

Wider C, Pollo C, Bloch J, Burkhard PR, Vingerhoets FJG. Long-term outcome of 50 consecutive Parkinson's disease patients treated with subthalamic deep brain stimulation. Parkinsonism Relat Disord 2008;14:114-9.

Wolters $\mathrm{CH}$, Anwander A, Tricoche X, Lew S, Johnson CR. Influence of local and remote white matter conductivity anisotropy for a thalamic source on EEG/MEC field and return current computation. Int J Bioelectromagn 2005;7:203-6.

Wolters CH, Anwander A, Tricoche X, Weinstein D, Koch MA, MacLeod RS. Influence of tissue conductivity anisotropy on EEG/MEG field and return current computation in a realistic head model: a simulation and visualization study using high-resolution finite element modeling. Neuroimage 2006;30:813-26.

Wolters CH, Köstler H, Möller C, Härtlein J, Grasedyck L, Hackbusch W. Numerical mathematics of the subtraction method for the modeling of a current dipole in EEG source reconstruction using finite element head models. SIAM J Sci Comput 2007:30:24-45.

Yousif N, Bayford R, Bain PG, Liu X. The peri-electrode space is a significant element of the electrode-brain interface in deep brain stimulation: a computational study. Brain Res Bull 2007;74:361-8. 